\title{
Chapter 7 \\ The Neurochemistry of Alzheimer's \\ Disease: One of the Most Common Causes \\ of Reduced Capability in the Adult \\ Population
}

\author{
Kaj Blennow and Henrik Zetterberg
}

\subsection{Introduction}

Alzheimer's disease (AD) is the most common neurodegenerative disease and a major cause of capability impairment in the elderly. The first detectable pathology, which occurs decades before clinical symptom onset, is the accumulation of extracellular amyloid plaques in the brain, which have as their core the 42-amino-acidlong amyloid $\beta(\mathrm{A} \beta)$ protein. Biomarker studies suggest that $\mathrm{A} \beta$ accumulation is followed by increased phosphorylation and secretion of tau, a microtubuleassociated axonal protein that is highly expressed in cortical neurons. Dysfunctional tau metabolism leads to AD-type neurodegeneration, with the development of dystrophic neurites and intraneuronal neurofibrillary tangles that are composed of

\footnotetext{
K. Blennow ( $\square)$

Department of Psychiatry and Neurochemistry, Institute of Neuroscience \& Physiology, The Sahlgrenska Academy at the University of Gothenburg, Gothenburg, Sweden

Clinical Neurochemistry Laboratory, Sahlgrenska University Hospital, Mölndal, Sweden

Centre for Ageing and Health - AgeCap, University of Gothenburg,

Gothenburg, Sweden

e-mail: kaj.blennow@neuro.gu.se

H. Zetterberg

Department of Psychiatry and Neurochemistry, Institute of Neuroscience \& Physiology,

The Sahlgrenska Academy at the University of Gothenburg, Gothenburg, Sweden

Clinical Neurochemistry Laboratory, Sahlgrenska University Hospital, Mölndal, Sweden

Centre for Ageing and Health - AgeCap, University of Gothenburg,

Gothenburg, Sweden

Department of Neurodegenerative Disease, UCL Institute of Neurology, London, UK

UK Dementia Research Institute at UCL, London, UK

e-mail: henrik.zetterberg@clinchem.gu.se
} 
hyperphosphorylated and truncated tau proteins. Neurodegeneration translates into the AD clinical syndrome, with neurodegeneration being spatially and temporally associated with the pattern of cognitive deficits that worsen as the disease progresses. Exactly what initiates the amyloidogenic cascade is not known and may well be multifactorial, but the major risk factors are increasing age and the $\varepsilon 4$ allele of the apolipoprotein $\mathrm{E}(A P O E)$ gene, which is associated with a major increase in $\mathrm{AD}$ risk.

\subsubsection{The Molecular Neuropathology of AD}

The key microscopic neuropathological features of AD include neuronal and synaptic degeneration, accompanied by different forms of protein aggregates called plaques, neurofibrillary tangles and neuropil threads, Hirano bodies, and granulovacuolar degeneration (Perry, 1986; Terry \& Davies, 1980; Tomlinson et al., 1968, 1970). Plaques and tangles are found in the hippocampus and cortex, as well as in subcortical grey matter nuclei, such as the nucleus basalis of Meynert and locus coeruleus (Mann et al., 1985; Tomlinson, 1989), while Hirano bodies and granulovacuolar degeneration are largely restricted to the hippocampus. Plaques and tangles are found with increasing frequency with ageing, including in cognitively unimpaired individuals, and become very common at higher ages (Mann et al., 1984, 1985; Tomlinson, 1989; Dayan, 1970; Mann et al., 1984; Price et al., 2009).

Plaques are primarily composed of aggregated $\beta$-amyloid (A $\beta)$, and are rounded extracellular lesions (Masters et al., 1985; Glenner \& Wong, 1984) (Fig. 7.1). The majority of $\mathrm{A} \beta$ found in plaques ends at position $42(\mathrm{~A} \beta 42)$, which is a form of $\mathrm{A} \beta$ that is most prone to aggregation, in contrast to $\mathrm{A} \beta 40$, which is the most abundant variant. Plaques exist as diffuse plaques that are regarded as being the earliest form, and neuritic plaques, which consist of a central core of amyloid fibres surrounded

Fig. 7.1 Histology of amyloid plaques and tau pathology in Alzheimer's disease. Plaques (green arrow) are extracellular deposits of $\beta$-amyloid $(\mathrm{A} \beta)$ surrounded by dystrophic neuritis, while tangles (red arrow) and neuropil threads (blue arrow) are composed abnormally phosphorylated tau protein

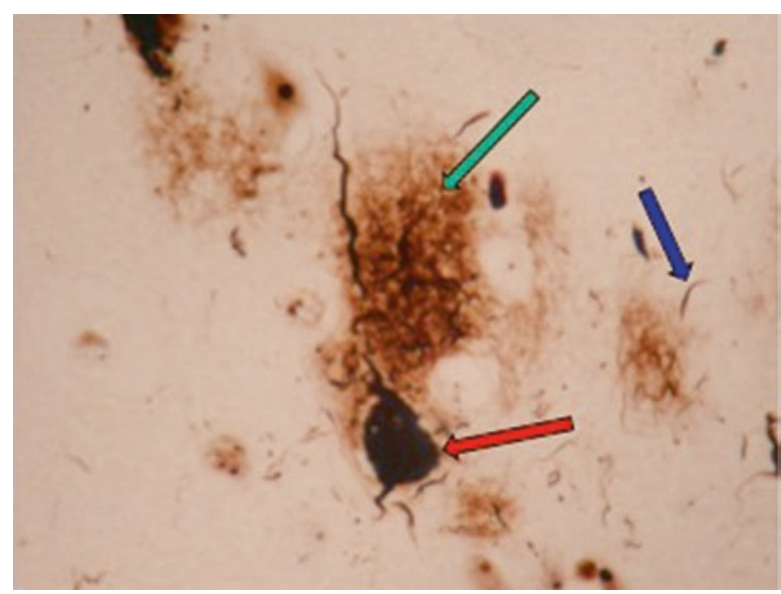


by dystrophic neurites filled with fibrillary tau protein, and reactive astrocytes and microglia (Thal et al., 2002). Tangles are located in the neuronal cytoplasm, while neuropil threads are extended structures in the neuropil (Fig. 7.1); both are primarily formed of hyperphosphorylated tau protein (Grundke-Iqbal et al., 1986).

In elderly patients with $\mathrm{AD}$, the number of plaques and tangles varies considerably and overlaps with the number found in cognitively unimpaired elderly people (Mann et al., 1984, 1985; Hansen et al., 1988). A common explanation for this overlap is that elderly patients with AD-type dementia have other pathologies in addition to plaques and tangles, including $\alpha$-synuclein and TDP-43 deposits, as well as cerebrovascular disease and other pathologies (James et al., 2016; Kovacs et al., 2013). Other frequent points of discussion are whether certain individuals may be more or less susceptible to AD-type pathological changes, whether lifestyle affects the risk or phenotypic expression of the pathologies and whether lifestyle changes could influence the risk of developing dementia from AD. Positive results for multimodal lifestyle interventions have been reported (Ngandu et al., 2015), but an outstanding question is whether such interventions have a direct impact on the neuropathology of $\mathrm{AD}$ (a recent biomarker-based study suggests that they do not (Steen Jensen et al., 2016)), or whether they confer protection via ancillary mechanisms such as improved cerebral blood flow, myelination, increased synaptic density or improved immune function. In any case, studies with long-term clinical follow-up suggest that $>90 \%$ of plaque-positive individuals eventually develop dementia (Buchhave et al., 2012; Rowe et al., 2013), indicating that biomarkers for plaque pathology can be used to identify AD in the preclinical stage of the disease, and thus may have value as tools to enrich the search for asymptomatic AD in intervention studies.

\subsection{Clinical Neurochemistry and Fluid Biomarkers}

The development of cerebrospinal fluid (CSF) and blood (plasma and serum) biomarkers is a lead project for the Clinical Neurochemistry Laboratory. Such biomarkers for the different types of pathophysiology found in the brains of elderly people have several applications, both in research and in the clinic. Firstly, biomarkers have value in the clinical diagnosis, especially in the early stages of the disease, when symptoms are vague and uncharacteristic. Secondly, biomarkers are key in clinical research to deepen our understanding of the pathogenesis of the disease, for example the time course of different pathologies, how they contribute to the symptoms, how lifestyle and other risk factors may influence the risk of pathological changes, how pathologies may cause subtle changes in behaviour that are not classifiable by traditional clinical criteria and time trends in pathology prevalence, incidence and phenotypic expression (Blennow et al., 2010).

For research on $\mathrm{AD}$ and age-related cognitive decline, several highly validated and specific biomarkers are available. Imaging biomarkers include both amyloid and tau positron emission tomography (PET), which give a measure of the density 
of aggregated amyloid and tau deposits in the brain, and magnetic resonance imaging (MRI), which gives a measure of total and regional brain volume, and thereby degree of neuronal loss. The CSF biomarkers total tau (T-tau), phosphorylated tau (P-tau) and the 42-amino-acid isoform of $\beta$-amyloid (A $\beta 42)$ reflect the intensity of neuronal and axonal degeneration, the phosphorylation state of tau and tangle formation, and the aggregation and deposition of the A $\beta 42$ peptide into plaques (Blennow \& Hampel, 2003). These core AD CSF biomarkers are reviewed below.

\subsubsection{The Core CSF Biomarkers for AD}

Using enzyme-linked immunosorbent assay (ELISA) methods for T-tau that measure all tau isoforms irrespective of phosphorylation state (Blennow et al., 1995), tau phosphorylated at threonine 181 (P-tau181) (Vanmechelen et al., 2000), and $\beta$-amyloid 1-42 (A $\beta 42)$ (Andreasen et al. 1999a, b), a very large number of publications have consistently shown that most patients with a clinical diagnosis of AD show the typical 'AD biomarker profile', consisting of increased T-tau and P-tau, together with decreased A $\beta 42$ (Olsson et al., 2016). In line with this, studies in which the diagnosis has been confirmed at autopsy have shown even better diagnostic performance in identifying AD dementia (Koopman et al., 2009; Shaw et al., 2009).

Given that clinical diagnosis is most difficult in the earlier stages of the disease, it is important that $\mathrm{AD}$ biomarkers also have high diagnostic accuracy in the mild cognitive impairment (MCI) stage of the disease. MCI is a heterogeneous syndrome that can have many causes; only around $50 \%$ of MCI patients will progress to AD dementia. A first study evaluating whether the combination of high tau and low $\mathrm{A} \beta 42$ in CSF can predict which MCI patients will progress to AD dementia showed that these core AD CSF biomarkers are already positive at this early clinical stage (Andreasen et al., 1999a, b). Furthermore, the CSF biomarkers had similar concentrations in a new CSF sample taken when patients had reached the dementia stage (Andreasen et al., 1999a, b), suggesting that these CSF biomarkers have already plateaued during the MCI stage, and will not change further during the transition stage.

In 2006, the first study with an extended clinical follow-up, which is needed to ascertain which MCI will not progress to AD dementia, showed that, while cognitively stable MCI cases had normal biomarkers, 95\% of MCI patients who progressed to AD dementia had the typical AD CSF biomarker (Hansson et al., 2006). This very high capacity of the core AD CSF biomarkers to identify progressive MCI patients (whom we today call prodromal AD) has been verified by a number of large multicentre studies in Europe (Visser et al., 2009) and the USA (Shaw et al., 2009), and in the Swedish Brain Power study (Mattsson et al., 2009). 


\subsubsection{CSF Biomarkers in Preclinical AD}

An important question for the application of CSF biomarkers in population-based studies is whether they can detect AD pathophysiology in the subset of cognitively unimpaired elderly who have clinically silent pathology. Indeed, in 2003 a first study showed that cognitively unimpaired 85-year-olds who at clinical follow-up had developed $\mathrm{AD}$ dementia already had decreased CSF A $\beta 42$ levels in this preclinical stage, while both CSF T-tau and P-tau were normal (Skoog et al., 2003). This finding suggests that a CSF test can identify brain amyloid deposition, the central type of AD pathology, preclinically, a finding that was also confirmed in subsequent population-based cohorts of cognitively unimpaired elderly of less advanced age (70-78 years), and even earlier; in a sample taken up to eight years before the appearance of symptoms (Gustafson et al., 2007). Furthermore, these findings support the hypothesis that amyloid deposition (low CSF A $\beta 42$ ) is present before either neurodegeneration (high T-tau) or tau pathology (high P-tau) is evident. This will facilitate projects examining risk factors for $\mathrm{A} \beta$ and tau pathology (and not just their clinical expression) using a molecular epidemiology approach, as discussed in detail below.

\subsubsection{New Biomarkers to Monitor Synaptic Dysfunction}

Synaptic dysfunction or degeneration is another key feature of AD pathophysiology (Davidsson \& Blennow, 1990). Synaptic degeneration correlates more closely with the severity of cognitive disturbances than amyloid plaque load (Terry et al., 1991; DeKosky \& Scheff, 1990). Recent studies suggest that early amyloid aggregates, including soluble A $\beta$ oligomers (Shankar et al., 2007) and intracellular A $\beta$ accumulations (Zou et al., 2015), may be the driver behind the synaptic dysfunction with dendritic spine loss in AD.

Combining the core CSF biomarkers for AD with reliable biomarkers to monitor synaptic and dendritic function in longitudinal clinical and epidemiological studies may give clues about AD pathogenesis, specifically the temporal evolution of the different forms of AD pathophysiology, as well as their interrelations, risk factors and relative contributions to cognitive symptoms.

One candidate biomarker for synaptic dysfunction is neurogranin, a dendritic protein expressed in the cortex and hippocampus, which is specifically located in dendritic spines (Represa et al., 1990; Guadano-Ferraz et al., 2005). Neurogranin is involved in synaptic plasticity and the induction of long-term potentiation (LTP) in the hippocampus (Fedorov et al., 1995; Chen et al., 1997), and thus of interest in disorders involving memory dysfunction. In the normal brain, neurogranin expression is highest in the association cortex, while lower neurogranin levels are seen in the cortex and hippocampus of the AD brain (Davidsson \& Blennow 1990; Reddy et al., 2005), probably as a consequence of synaptic loss. 
A first pilot study on CSF neurogranin as a biomarker for synaptic degeneration in $\mathrm{AD}$ found a noticeable increase as compared with cognitively unimpaired individuals of similar age (Thorsell et al., 2010), a finding that could be confirmed in the first study measuring CSF neurogranin by ELISA (Kvartsberg et al., 2015a). High CSF neurogranin in both AD dementia and prodromal AD has subsequently been confirmed in many large studies (Hellwig et al., 2015; Kvartsberg et al., 2015b), including the Alzheimer's Disease Neuroimaging Initiative (ADNI) multicentre study (Portelius et al., 2015). Interestingly, high CSF neurogranin correlates with future rate of neuronal loss and reductions in cortical metabolism (Portelius et al., 2015). Furthermore, increased CSF neurogranin appears to be specific to AD, since normal levels are found in many other neurodegenerative disorders, e.g., frontotemporal dementia, Lewy body dementia and progressive supranuclear palsy (Wellington et al., 2016).

\subsection{The Need for Molecular Epidemiology Studies}

The vast majority of population-based studies on AD risk profiling have not classified patients or controls using biomarkers reflecting specific AD pathophysiologies, i.e., amyloid and tau pathology (Yu et al., 2020), but instead have examined risk factors for age-related dementia or clinically diagnosed 'probable AD'. Such studies are also hampered by the complex pathophysiology of late-onset AD and the difficulties in making a correct clinical diagnosis. This introduces the risk that a proportion of cognitively unimpaired individuals (classified as control subjects) have preclinical AD pathology and thus limits the ability to correctly identify the underlying type of pathology (AD or other age-related dementias). Indeed, type II diabetes has been found to be a risk factor for 'probable AD' (defined as an 'amnestic dementia') in studies without biomarkers (Lu et al., 2009), while clinical studies validating clinical associations with pathology at autopsy show that the basis for the association between diabetes and cognitive disturbances is through cerebrovascular lesions, and not amyloid plaques or tau pathology (Abner et al., 2016; Nelson et al., 2011). In agreement, population-based studies using biomarkers have found that mid-life risk factors previously claimed to be associated with dementia (or 'probable AD'), such as obesity, smoking, diabetes, hypertension and cardiac disease were not associated with amyloid pathology, but with neurodegeneration (Vemuri et al., 2012). Similarly, GWAS studies have found an association between gene loci and clinically diagnosed 'probable AD', but when these loci are examined in cohorts with autopsy-verified diagnoses, many loci show no association with either amyloid plaques or tau pathology, but with cerebrovascular lesions (Beecham et al., 2014). Conversely, genetic studies using biomarkers for $\mathrm{A} \beta$ and tau pathologies as endophenotypes identify slightly different sets of susceptibility genes (Deming et al., 2017), and the association of AD with APOE \&4 grows stronger (Andreasson et al., 2014). 
In addition, it may be of interest to link modifiable risk factors to specific pathophysiologies following the amyloid (A), tau (T) and neurodegeneration (N) classification system (Jack Jr et al., 2016), in order to understand which risk factors are directly associated with $\mathrm{AD}$, and which are associated with old-age dementia in general.

\subsection{Blood Tests will Facilitate the Implementation of Biomarkers in Population-Based Studies}

While PET imaging and CSF biomarkers are well-validated tools to measure AD pathophysiology, they have some drawbacks that may make them more difficult to implement in epidemiological studies. PET imaging is expensive and has limited accessibility, and CSF collection by lumbar puncture may be frightening for study participants and regarded as complicated and time-consuming by many clinicians. Thus, the ability to measure proteins linked to AD pathophysiology in blood samples may facilitate the implementation of biomarkers.

Recent technical developments have provided ultrasensitive techniques, such as immuno- magnetic reduction (IMR) and single-molecule array (Simoa), that enable the measurement of brain-derived proteins in blood samples (Andreasson et al., 2016).

\subsubsection{Brain Amyloidosis - Plasma Aß42/40 Ratio}

Plasma levels of amyloid $\beta$, specifically the $A \beta 42 / 40$ ratio, are reduced in Alzheimer's disease (AD) and show high concordance with brain amyloidosis as assessed by PET. The explanation is likely to be that the 42-amino-acid-long variant of amyloid $\beta$ (A $\beta 42)$, which is hydrophobic and prone to aggregate (Jarrett et al., 1993), is deposited in amyloid plaques, with a smaller amount being secreted to the CSF (Olsson et al., 2016) and blood. Using the ultrasensitive Simoa technology to measure $A \beta 42$ in plasma (Zetterberg et al., 2011), a statistically significant correlation was found between $\mathrm{A} \beta 42$ (and the $\mathrm{A} \beta 42 / 40$ ratio) in plasma and CSF, as well as with amyloid load measured by PET technology (Janelidze et al., 2016). Plasma $\mathrm{A} \beta 42$ and $\mathrm{A} \beta 40$ can also be measured using fully automated instruments (Palmqvist et al., 2019), and mass spectrometry techniques (Schindler et al., 2019), showing high concordance with amyloid PET measures. 


\subsubsection{Tau Pathology - Phosphorylated Tau}

Tau phosphorylated at position 181 (P-tau181) can be measured in plasma using MSD technology; a first paper showed a marked increase in AD and a clear association with tau PET (Mielke et al., 2018), a finding that was validated in two large clinical cohorts (Janelidze et al., 2020; Thijssen et al., 2020). Importantly, plasma P-tau181 was normal in other tau-pathologies, including frontotemporal dementia, progressive supranuclear palsy and corticobasal degeneration (Thijssen et al., 2020). Another paper presented a method for measuring plasma P-tau181 using the Simoa technique, and showed a marked increase in $\mathrm{AD}$, a clear association with tau pathology measured by PET, but also that plasma P-tau was already increased in individuals with brain amyloidosis, but still a negative tau PET scan (Karikari et al., 2020). Other tau variants, specifically P-tau217, are also measurable in blood samples, with very promising results (Palmqvist et al., 2020). Taken together, these studies indicate that plasma P-tau is a reliable biomarker for tau pathology, which is easily accessible.

\subsubsection{Neurodegeneration - Neurofilament Light Protein}

Total tau (T-tau) and neurofilament light (NFL) reflect neuronal injury and are measurable in blood. Both these biomarkers show promise as a biomarker for acute neuronal injury (e.g., acute traumatic or hypoxic brain injury), with rapid and dramatic increases that also predict clinical outcome (Moseby-Knappe et al., 2019). However, T-tau in blood only shows a marginal increase in $\mathrm{AD}$, but still correlates with atrophy measured by MRI and reduced cortical metabolism assessed by FDGPET (Mattsson et al., 2016).

Another neuronal protein, neurofilament light (NFL), can also be measured in blood samples using the Simoa technique (Kuhle et al., 2016). Similar to tau protein, plasma NFL concentrations are increased in both AD dementia and MCI cases (Mattsson et al., 2017). Interestingly, higher plasma NFL concentrations were associated with amyloid deposition (as evaluated by amyloid PET), and predicted progression of cognitive decline and brain atrophy (Mattsson et al., 2017). However, a robust increase in blood NFL levels is found in several neurodegenerative disorders, not only in $\mathrm{AD}$, indicating that it is a non-disease-specific biomarker for axonal degeneration (Lewczuk et al., 2018). Importantly, plasma NFL is already increasing in the preclinical phase of AD (Weston et al., 2017; Preische et al., 2019). These findings support the idea that plasma NFL can be used in the epidemiology setting as a neurodegeneration marker.

In summary, we have witnessed a rapid and successful development of blood biomarkers for specific pathologies in AD, including brain amyloidosis (A $\beta 42 / 40$ ratio), tau pathology (P-tau), and neurodegeneration (NFL). The implementation of blood biomarkers in epidemiological studies may foster the understanding of 
lifestyle risk factors for $\mathrm{AD}$, and repeated blood sampling may prove useful to assess the benefits of lifestyle interventions.

\section{References}

Abner, E. L., Nelson, P. T., Kryscio, R. J., Schmitt, F. A., Fardo, D. W., Woltjer, R. L., et al. (2016). Diabetes is associated with cerebrovascular but not Alzheimer's disease neuropathology. Alzheimer's \& Dementia: The Journal of the Alzheimer's Association, 12(8), 882-889.

Andreasen, N., Minthon, L., Clarberg, A., Davidsson, P., Gottfries, J., Vanmechelen, E., et al. (1999a). Sensitivity, specificity, and stability of CSF-tau in AD in a community-based patient sample. Neurology, 53(7), 1488-1494.

Andreasen, N., Minthon, L., Vanmechelen, E., Vanderstichele, H., Davidsson, P., Winblad, B., et al. (1999b). Cerebrospinal fluid tau and Abeta42 as predictors of development of Alzheimer's disease in patients with mild cognitive impairment. Neuroscience Letters, 273(1), 5-8.

Andreasson, U., Lautner, R., Schott, J. M., Mattsson, N., Hansson, O., Herukka, S. K., et al. (2014). CSF biomarkers for Alzheimer's pathology and the effect size of APOE varepsilon4. Molecular Psychiatry, 19(2), 148-149.

Andreasson, U., Blennow, K., \& Zetterberg, H. (2016). Update on ultrasensitive technologies to facilitate research on blood biomarkers for central nervous system disorders. Alzheimers Dement (Amst), 3, 98-102.

Beecham, G. W., Hamilton, K., Naj, A. C., Martin, E. R., Huentelman, M., Myers, A. J., et al. (2014). Genome-wide association meta-analysis of neuropathologic features of Alzheimer's disease and related dementias. PLoS Genetics, 10(9), e1004606.

Blennow, K., \& Hampel, H. (2003). CSF markers for incipient Alzheimer's disease. Lancet Neurology, 2(10), 605-613.

Blennow, K., Wallin, A., Agren, H., Spenger, C., Siegfried, J., \& Vanmechelen, E. (1995). Tau protein in cerebrospinal fluid: a biochemical marker for axonal degeneration in Alzheimer disease? Molecular and Chemical Neuropathology, 26(3), 231-245.

Blennow, K., Hampel, H., Weiner, M., \& Zetterberg, H. (2010). Cerebrospinal fluid and plasma biomarkers in Alzheimer disease. Nature Reviews Neurology, 6(3), 131-144.

Buchhave, P., Minthon, L., Zetterberg, H., Wallin, A. K., Blennow, K., \& Hansson, O. (2012). Cerebrospinal fluid levels of beta-amyloid 1-42, but not of tau, are fully changed already 5 to 10 years before the onset of Alzheimer dementia. Archives of General Psychiatry, 69(1), 98-106.

Chen, S. J., Sweatt, J. D., \& Klann, E. (1997). Enhanced phosphorylation of the postsynaptic protein kinase $\mathrm{C}$ substrate $\mathrm{RC} 3 /$ neurogranin during long-term potentiation. Brain Research, 749(2), 181-187.

Davidsson, P., \& Blennow, K. (1990). Neurochemical dissection of synaptic pathology in Alzheimer's disease. International Psychogeriatrics / IPA, 10(1), 11-23.

Dayan, A. D. (1970). Quantitative histological studies on the aged human brain. I. Senile plaques and neurofibrillary tangles in 'normal' patients. Acta Neuropathologica, 16(2), 85-94.

DeKosky, S. T., \& Scheff, S. W. (1990). Synapse loss in frontal cortex biopsies in Alzheimer's disease: correlation with cognitive severity. Annals of Neurology, 27(5), 457-464.

Deming, Y., Li, Z., Kapoor, M., Harari, O., Del-Aguila, J. L., Black, K., et al. (2017). Genomewide association study identifies four novel loci associated with Alzheimer's endophenotypes and disease modifiers. Acta Neuropathologica, 133(5), 839-856.

Fedorov, N. B., Pasinelli, P., Oestreicher, A. B., DeGraan, P. N., \& Reymann, K. G. (1995). Antibodies to postsynaptic PKC substrate neurogranin prevent long-term potentiation in hippocampal CA1 neurons. The European Journal of Neuroscience, 7(4), 819-822. 
Glenner, G. G., \& Wong, C. W. (1984). Alzheimer's disease: initial report of the purification and characterization of a novel cerebrovascular amyloid protein. Biochemical and Biophysical Research Communications, 120(3), 885-890.

Grundke-Iqbal, I., Iqbal, K., Tung, Y. C., Quinlan, M., Wisniewski, H. M., \& Binder, L. I. (1986). Abnormal phosphorylation of the microtubule-associated protein tau (tau) in Alzheimer cytoskeletal pathology. Proceedings of the National Academy of Sciences of the United States of America, 83(13), 4913-4917.

Guadano-Ferraz, A., Vinuela, A., Oeding, G., Bernal, J., \& Rausell, E. (2005). RC3/neurogranin is expressed in pyramidal neurons of motor and somatosensory cortex in normal and denervated monkeys. Journal of Comparative Neurology, 493(4), 554-570.

Gustafson, D. R., Skoog, I., Rosengren, L., Zetterberg, H., \& Blennow, K. (2007). Cerebrospinal fluid beta-amyloid 1-42 concentration may predict cognitive decline in older women. Journal of Neurology, Neurosurgery, and Psychiatry, 78(5), 461-464.

Hansen, L. A., Deteresa, R., Tobias, H., Alford, M., \& Terry, R. D. (1988). Neocortical morphometry and cholinergic neurochemistry in Pick's disease. The American Journal of Pathology, 131(3), 507-518.

Hansson, O., Zetterberg, H., Buchhave, P., Londos, E., Blennow, K., \& Minthon, L. (2006). Association between CSF biomarkers and incipient Alzheimer's disease in patients with mild cognitive impairment: a follow-up study. Lancet Neurology, 5(3), 228-234.

Hellwig, K., Kvartsberg, H., Portelius, E., Andreasson, U., Oberstein, T. J., Lewczuk, P., et al. (2015). Neurogranin and YKL-40: independent markers of synaptic degeneration and neuroinflammation in Alzheimer's disease. Alzheimer's Research \& Therapy, 7, 74.

Jack, C. R., Jr., Bennett, D. A., Blennow, K., Carrillo, M. C., Feldman, H. H., Frisoni, G. B., et al. (2016). A/T/N: An unbiased descriptive classification scheme for Alzheimer disease biomarkers. Neurology, 87(5), 539-547.

James, B. D., Wilson, R. S., Boyle, P. A., Trojanowski, J. Q., Bennett, D. A., \& Schneider, J. A. (2016). TDP-43 stage, mixed pathologies, and clinical Alzheimer's-type dementia. Brain, 139(11), 2983-2993.

Janelidze, S., Stomrud, E., Palmqvist, S., Zetterberg, H., van Westen, D., Jeromin, A., et al. (2016). Plasma beta-amyloid in Alzheimer's disease and vascular disease. Scientific Reports, 6, 26801.

Janelidze, S., Mattsson, N., Palmqvist, S., Smith, R., Beach, T. G., Serrano, G. E., et al. (2020). Plasma P-tau181 in Alzheimer's disease: relationship to other biomarkers, differential diagnosis, neuropathology and longitudinal progression to Alzheimer's dementia. Nature Medicine, 26(3), 379-386.

Jarrett, J. T., Berger, E. P., \& Lansbury, P. T., Jr. (1993). The carboxy terminus of the beta amyloid protein is critical for the seeding of amyloid formation: implications for the pathogenesis of Alzheimer's disease. Biochemistry, 32(18), 4693-4697.

Karikari, T. K., Pascoal, T. A., Ashton, N. J., Janelidze, S., Benedet, A. L., Rodriguez, J. L., et al. (2020). Blood phosphorylated tau 181 as a biomarker for Alzheimer's disease: a diagnostic performance and prediction modelling study using data from four prospective cohorts. Lancet Neurology, 19(5), 422-433.

Koopman, K., Le Bastard, N., Martin, J. J., Nagels, G., De Deyn, P. P., \& Engelborghs, S. (2009). Improved discrimination of autopsy-confirmed Alzheimer's disease (AD) from non-AD dementias using CSF P-tau(181P). Neurochemistry International, 55(4), 214-218.

Kovacs, G. G., Milenkovic, I., Wohrer, A., Hoftberger, R., Gelpi, E., Haberler, C., et al. (2013). Non-Alzheimer neurodegenerative pathologies and their combinations are more frequent than commonly believed in the elderly brain: a community-based autopsy series. Acta Neuropathologica, 126(3), 365-384.

Kuhle, J., Barro, C., Andreasson, U., Derfuss, T., Lindberg, R., Sandelius, A., et al. (2016). Comparison of three analytical platforms for quantification of the neurofilament light chain in blood samples: ELISA, electrochemiluminescence immunoassay and Simoa. Clinical Chemistry and Laboratory Medicine, 54(10), 1655-1661. 
Kvartsberg, H., Duits, F. H., Ingelsson, M., Andreasen, N., Ohrfelt, A., Andersson, K., et al. (2015a). Cerebrospinal fluid levels of the synaptic protein neurogranin correlates with cognitive decline in prodromal Alzheimer's disease. Alzheimers Dement, 11(10), 1180-1190.

Kvartsberg, H., Portelius, E., Andreasson, U., Brinkmalm, G., Hellwig, K., Lelental, N., et al. (2015b). Characterization of the postsynaptic protein neurogranin in paired cerebrospinal fluid and plasma samples from Alzheimer's disease patients and healthy controls. Alzheimer's Research \& Therapy, 7(1), 40.

Lewczuk, P., Riederer, P., O'Bryant, S. E., Verbeek, M. M., Dubois, B., Visser, P. J., et al. (2018). Cerebrospinal fluid and blood biomarkers for neurodegenerative dementias: an update of the consensus of the Task Force on Biological Markers in Psychiatry of the World Federation of Societies of Biological Psychiatry. The World Journal of Biological Psychiatry, 19(4), 244-328.

Lu, F. P., Lin, K. P., \& Kuo, H. K. (2009). Diabetes and the risk of multi-system aging phenotypes: a systematic review and meta-analysis. PLoS One, 4(1), e4144.

Mann, D. M., Yates, P. O., \& Marcyniuk, B. (1984). Alzheimer's presenile dementia, senile dementia of Alzheimer type and Down's syndrome in middle age form an age-related continuum of pathological changes. Neuropathology and Applied Neurobiology, 10(3), 185-207.

Mann, D. M., Yates, P. O., \& Marcyniuk, B. (1985). Some morphometric observations on the cerebral cortex and hippocampus in presenile Alzheimer's disease, senile dementia of Alzheimer type and Down's syndrome in middle age. Journal of the Neurological Sciences, 69(3), $139-159$.

Masters, C. L., Simms, G., Weinman, N. A., Multhaup, G., McDonald, B. L., \& Beyreuther, K. (1985). Amyloid plaque core protein in Alzheimer disease and Down syndrome. Proceedings of the National Academy of Sciences of the United States of America, 82(12), 4245-4249.

Mattsson, N., Zetterberg, H., Hansson, O., Andreasen, N., Parnetti, L., Jonsson, M., et al. (2009). CSF biomarkers and incipient Alzheimer disease in patients with mild cognitive impairment. JAMA: The Journal of the American Medical Association, 302(4), 385-393.

Mattsson, N., Zetterberg, H., Janelidze, S., Insel, P. S., Andreasson, U., Stomrud, E., et al. (2016). Plasma tau in Alzheimer disease. Neurology, 87(17), 1827-1835.

Mattsson, N., Andreasson, U., Zetterberg, H., \& Blennow, K. (2017). Alzheimer's disease neuroimaging I. Association of plasma neurofilament light with neurodegeneration in patients with Alzheimer disease. JAMA Neurology, 74(5), 557-566.

Mielke, M. M., Hagen, C. E., Xu, J., Chai, X., Vemuri, P., Lowe, V. J., et al. (2018). Plasma phospho-tau181 increases with Alzheimer's disease clinical severity and is associated with tauand amyloid-positron emission tomography. Alzheimers Dement, 14(8), 989-997.

Moseby-Knappe, M., Mattsson, N., Nielsen, N., Zetterberg, H., Blennow, K., Dankiewicz, J., et al. (2019). Serum neurofilament light chain for prognosis of outcome after cardiac arrest. JAMA Neurology, 76(1), 64-71.

Nelson, P. T., Head, E., Schmitt, F. A., Davis, P. R., Neltner, J. H., Jicha, G. A., et al. (2011). Alzheimer's disease is not 'brain aging': neuropathological, genetic, and epidemiological human studies. Acta Neuropathologica, 121(5), 571-587.

Ngandu, T., Lehtisalo, J., Solomon, A., Levalahti, E., Ahtiluoto, S., Antikainen, R., et al. (2015). A 2-year multidomain intervention of diet, exercise, cognitive training, and vascular risk monitoring versus control to prevent cognitive decline in at-risk elderly people (FINGER): a randomised controlled trial. Lancet, 385(9984), 2255-2263.

Olsson, B., Lautner, R., Andreasson, U., Ohrfelt, A., Portelius, E., Bjerke, M., et al. (2016). CSF and blood biomarkers for the diagnosis of Alzheimer's disease: a systematic review and metaanalysis. Lancet Neurology, 15(7), 673-684.

Palmqvist, S., Janelidze, S., Stomrud, E., Zetterberg, H., Karl, J., Zink, K., et al. (2019). Performance of fully automated plasma assays as screening tests for Alzheimer disease-related beta-amyloid status. JAMA Neurology, 76(9), 1060-1069.

Palmqvist, S., Janelidze, S., Quiroz, Y. T., Zetterberg, H., Lopera, F., Stomrud, E., et al. (2020). Discriminative accuracy of plasma phospho-tau 217 for Alzheimer disease vs other neurodegenerative disorders. JAMA: The Journal of the American Medical Association, 324(8), 772-781. 
Perry, R. H. (1986). Recent advances in neuropathology. British Medical Bulletin, 42(1), 34-41.

Portelius, E., Zetterberg, H., Skillback, T., Tornqvist, U., Andreasson, U., Trojanowski, J. Q., et al. (2015). Cerebrospinal fluid neurogranin: relation to cognition and neurodegeneration in Alzheimer's disease. Brain: A Journal of Neurology, 138(Pt 11), 3373-3385.

Preische, O., Schultz, S. A., Apel, A., Kuhle, J., Kaeser, S. A., Barro, C., et al. (2019). Serum neurofilament dynamics predicts neurodegeneration and clinical progression in presymptomatic Alzheimer's disease. Nature Medicine, 25(2), 277-283.

Price, J. L., McKeel, D. W., Jr., Buckles, V. D., Roe, C. M., Xiong, C., Grundman, M., et al. (2009). Neuropathology of nondemented aging: presumptive evidence for preclinical Alzheimer disease. Neurobiology of Aging, 30(7), 1026-1036.

Reddy, P. H., Mani, G., Park, B. S., Jacques, J., Murdoch, G., Whetsell, W., Jr., et al. (2005). Differential loss of synaptic proteins in Alzheimer's disease: implications for synaptic dysfunction. Journal of Alzheimer's Disease: JAD, 7(2), 103-117. discussion 73-80.

Represa, A., Deloulme, J. C., Sensenbrenner, M., Ben-Ari, Y., \& Baudier, J. (1990). Neurogranin: immunocytochemical localization of a brain-specific protein kinase $\mathrm{C}$ substrate. The Journal of Neuroscience, 10(12), 3782-3792.

Rowe, C. C., Bourgeat, P., Ellis, K. A., Brown, B., Lim, Y. Y., Mulligan, R., et al. (2013). Predicting Alzheimer disease with beta-amyloid imaging: results from the Australian imaging, biomarkers, and lifestyle study of ageing. Annals of Neurology, 74(6), 905-913.

Schindler, S. E., Bollinger, J. G., Ovod, V., Mawuenyega, K. G., Li, Y., Gordon, B. A., et al. (2019). High-precision plasma beta-amyloid 42/40 predicts current and future brain amyloidosis. Neurology, 93(17), e1647-e1e59.

Shankar, G. M., Bloodgood, B. L., Townsend, M., Walsh, D. M., Selkoe, D. J., \& Sabatini, B. L. (2007). Natural oligomers of the Alzheimer amyloid-beta protein induce reversible synapse loss by modulating an NMDA-type glutamate receptor-dependent signaling pathway. The Journal of Neuroscience, 27(11), 2866-2875.

Shaw, L. M., Vanderstichele, H., Knapik-Czajka, M., Clark, C. M., Aisen, P. S., Petersen, R. C., et al. (2009). Cerebrospinal fluid biomarker signature in Alzheimer's disease neuroimaging initiative subjects. Annals of Neurology, 65(4), 403-413.

Skoog, I., Davidsson, P., Aevarsson, O., Vanderstichele, H., Vanmechelen, E., \& Blennow, K. (2003). Cerebrospinal fluid beta-amyloid 42 is reduced before the onset of sporadic dementia: a population-based study in 85-year-olds. Dementia and Geriatric Cognitive Disorders, 15(3), 169-176.

Steen Jensen, C., Portelius, E., Siersma, V., Hogh, P., Wermuth, L., Blennow, K., et al. (2016). Cerebrospinal fluid amyloid beta and tau concentrations are not modulated by 16 weeks of moderate- to high-intensity physical exercise in patients with Alzheimer disease. Dementia and Geriatric Cognitive Disorders, 42(3-4), 146-158.

Terry, R. D., \& Davies, P. (1980). Dementia of the Alzheimer type. Annual Review of Neuroscience, 3, 77-95.

Terry, R. D., Masliah, E., Salmon, D. P., Butters, N., DeTeresa, R., Hill, R., et al. (1991). Physical basis of cognitive alterations in Alzheimer's disease: synapse loss is the major correlate of cognitive impairment. Annals of Neurology, 30(4), 572-580.

Thal, D. R., Rub, U., Orantes, M., \& Braak, H. (2002). Phases of A beta-deposition in the human brain and its relevance for the development of AD. Neurology, 58(12), 1791-1800.

Thijssen, E. H., La Joie, R., Wolf, A., Strom, A., Wang, P., Iaccarino, L., et al. (2020). Diagnostic value of plasma phosphorylated tau181 in Alzheimer's disease and frontotemporal lobar degeneration. Nature Medicine, 26(3), 387-397.

Thorsell, A., Bjerke, M., Gobom, J., Brunhage, E., Vanmechelen, E., Andreasen, N., et al. (2010). Neurogranin in cerebrospinal fluid as a marker of synaptic degeneration in Alzheimer's disease. Brain Research, 1362, 13-22.

Tomlinson, B. E. (1989). Second Dorothy S. Russell memorial. The neuropathology of Alzheimer's diseaselecture. Neuropathology and Applied Neurobiology, 15(6), 491-512. 
Tomlinson, B. E., Blessed, G., \& Roth, M. (1968). Observations on the brains of non-demented old people. Journal of the Neurological Sciences, 7(2), 331-356.

Tomlinson, B. E., Blessed, G., \& Roth, M. (1970). Observations on the brains of demented old people. Journal of the Neurological Sciences, 11(3), 205-242.

Vanmechelen, E., Vanderstichele, H., Davidsson, P., Van Kerschaver, E., Van Der Perre, B., Sjogren, M., et al. (2000). Quantification of tau phosphorylated at threonine 181 in human cerebrospinal fluid: a sandwich ELISA with a synthetic phosphopeptide for standardization. Neuroscience Letters, 285(1), 49-52.

Vemuri, P., Lesnick, T. G., Przybelski, S. A., Knopman, D. S., Roberts, R. O., Lowe, V. J., et al. (2012). Effect of lifestyle activities on Alzheimer disease biomarkers and cognition. Annals of Neurology, 72(5), 730-738.

Visser, P. J., Verhey, F., Knol, D. L., Scheltens, P., Wahlund, L. O., Freund-Levi, Y., et al. (2009). Prevalence and prognostic value of CSF markers of Alzheimer's disease pathology in patients with subjective cognitive impairment or mild cognitive impairment in the DESCRIPA study: a prospective cohort study. Lancet Neurology, 8(7), 619-627.

Wellington, H., Paterson, R. W., Portelius, E., Tornqvist, U., Magdalinou, N., Fox, N. C., et al. (2016). Increased CSF neurogranin concentration is specific to Alzheimer disease. Neurology, 86(9), 829-835.

Weston, P. S. J., Poole, T., Ryan, N. S., Nair, A., Liang, Y., Macpherson, K., et al. (2017). Serum neurofilament light in familial Alzheimer disease: a marker of early neurodegeneration. Neurology, 89(21), 2167-2175.

Yu, J. T., Xu, W., Tan, C. C., Andrieu, S., Suckling, J., Evangelou, E., et al. (2020). Evidence-based prevention of Alzheimer's disease: systematic review and meta-analysis of 243 observational prospective studies and 153 randomised controlled trials. Journal of Neurology, Neurosurgery, and Psychiatry, 91(11), 1201-1209.

Zetterberg, H., Mortberg, E., Song, L., Chang, L., Provuncher, G. K., Patel, P. P., et al. (2011). Hypoxia due to cardiac arrest induces a time-dependent increase in serum amyloid beta levels in humans. PLoS One, 6(12), e28263.

Zou, C., Montagna, E., Shi, Y., Peters, F., Blazquez-Llorca, L., Shi, S., et al. (2015). Intraneuronal APP and extracellular Abeta independently cause dendritic spine pathology in transgenic mouse models of Alzheimer's disease. Acta Neuropathologica, 129(6), 909-920.

Open Access This chapter is licensed under the terms of the Creative Commons Attribution 4.0 International License (http://creativecommons.org/licenses/by/4.0/), which permits use, sharing, adaptation, distribution and reproduction in any medium or format, as long as you give appropriate credit to the original author(s) and the source, provide a link to the Creative Commons license and indicate if changes were made.

The images or other third party material in this chapter are included in the chapter's Creative Commons license, unless indicated otherwise in a credit line to the material. If material is not included in the chapter's Creative Commons license and your intended use is not permitted by statutory regulation or exceeds the permitted use, you will need to obtain permission directly from the copyright holder.

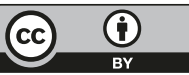

